Int. J. of Heat and Fluid Flow, Vol. 30(5), pp. 1016-1025, 2009

\title{
Large Eddy Simulations: how to evaluate resolution
}

\author{
Lars Davidson \\ Division of Fluid Dynamics, Department of Applied Mechanics, Chalmers University of \\ Technology, SE-412 96 Göteborg, Sweden
}

\begin{abstract}
The present paper gives an analysis of fully developed channel flow at Reynolds number of $R e=u_{\tau} \delta / \nu=4000$ based on the friction velocity, $u_{\tau}$, and half the channel height, $\delta$. Since the Reynolds number is high, the LES is coupled to a URANS model near the wall (hybrid LES-RANS) which acts as a wall model. It it found that the energy spectra is not a good measure of LES resolution; neither is the ratio of the resolved turbulent kinetic energy to the total one (i.e. resolved plus modelled turbulent kinetic energy). It is suggested that two-point correlations are the best measures for estimating LES resolution. It is commonly assumed that SGS dissipation takes place at high wavenumbers. Energy spectra of the fluctuating velocity gradients show that this is not true; the major part of the SGS dissipation takes place at low to midrange wavenumbers. Furthermore, the energy spectra of the fluctuating velocity gradients reveals that the accuracy of the predicted velocity
\end{abstract}

Email address: lada@chalmers.se (Lars Davidson)

URL: www.tfd.chalmers.se/ $11^{\sim}$ lada (Lars Davidson) 
gradients at the highest resolved wavenumbers is very poor.

Key words: LES, two-point correlations, energy spectra, dissipation spectra, SGS dissipation

\section{Introduction}

In wall-bounded Direct Numerical Simulations (DNS) and Large Eddy Simulations (LES) numerical experiments were carried out to determine the minimum required resolution to obtain accurate results (Moin and Kim, 1982; Piomelli, 1993; Piomelli and Chasnov, 1996; Moser et al., 1999; Hoyas and Jiménez, 2006). The grid resolution in DNS and wall-resolved LES is dictated by the necessity of resolving the energy-generating process high-speed inrushes and low-speed ejections (Robinson, 1991) in the viscous sub-layer and the buffer layer, often called the streak process. Since this process takes place in the viscous-dominating near-wall region, the required grid resolution must be expressed in inner variables, i.e. viscous units. In LES, the required grid resolution is $\Delta x^{+} \simeq 100, y^{+} \simeq 1$ (wall-adjacent cell centers) and $\Delta z^{+} \simeq 30$ where $x, y, z$ denote the streamwise, wall-normal and spanwise directions, respectively. Since a viscous length unit compared to the boundary layer thickness becomes smaller the higher the Reynolds number, the simulation times for DNS and wall-resolved LES at high Reynolds numbers become prohibitively high.

In the past ten years much research has been dedicated to the task of find- 
ing wall models that circumvent the requirement of defining the minimum near-wall cell size using inner variables. Spalart and his co-worker first proposed Detached-Eddy Simulations (Spalart et al., 1997; Travin et al., 2000), abbreviated as DES; later, different variants of hybrid LES/RANS models were proposed (Davidson and Peng, 2003; Temmerman et al., 2002; Tucker and Davidson, 2004; Hamba, 2003). In both DES and hybrid LES/RANS, the region near the walls is treated with Unsteady Reynolds-Averaged NavierStokes (URANS) and the outer region is treated by LES; the main difference is that, in DES, the entire boundary layer is treated with URANS whereas, in hybrid LES/RANS, the interface between URANS and LES is located in the inner part of the logarithmic region.

Another way to model the near-wall region is to use two-layer models (Balaras et al., 1996; Cabot, 1995; Cabot and Moin, 1999; Wang and Moin, 2002), also called "thin boundary layer equations". Here, the first node in the LES simulations is located - as when using wall functions - in the log-region. A new fine near-wall mesh is then created covering the walladjacent LES cell. Good results have been obtained for flow around a smooth three-dimensional hill (Tessicini et al., 2007), which is a fairly complex flow. Even wall functions - based on the instantaneous log-law - were found to give good results for this flow (Tessicini et al., 2007).

Assuming that the near-wall region is modelled using, for example, one 
of the approaches mentioned above, the question arises: how fine does the mesh need to be in the LES region? And, how do we, after having made an LES (assuming that there are no experimental data with which to compare), verify that the resolution was good enough? This is the focus of the present paper.

There are different ways to estimate the resolution of LES data. The first measure is probably to compare the modelled turbulence and stresses with the resolved ones. The smaller the ratio, the better the resolution. Another, similar way, is to compare the resolved turbulent kinetic energy to the modelled one. The energy spectra are commonly computed to find out whether they exhibit a $-5 / 3$ range and if they do the flow is considered to be well resolved. Another measure of the resolution may be to look at the two-point correlations to identify, for example, the ratio of the integral length scale to the cell size. A less common approach is to compare the SGS (i.e. modelled) dissipation due to fluctuating resolved strain-rates to that due to resolved or time-averaged strain-rates. In steady RANS, all SGS dissipation takes place due to time-averaged strain-rates whereas, in a wellresolved LES, most of the SGS dissipation occurs due to resolved fluctuations. It is commonly assumed that the SGS dissipation takes place at the highest resolved wavenumbers. This can be verified or disproved by making energy spectra of the SGS dissipation to find the wavenumbers at which the SGS 
dissipation does in fact take place.

The approaches mentioned above are used in the present paper to evaluate how relevant they are in estimating the resolution of LES simulations of fully developed channel flow. The Reynolds number is 4000 based on the friction velocity and the half-height of the channel. Five different resolutions are used. In order to be able to carry out LES at high Reynolds number, the near-wall region is covered by a wall model. In this work we have chosen to use hybrid LES/RANS.

The paper is organized as follows. The first three sub-sections present the equations, the turbulence model and the numerical method. The following section presents and analyzes the results using different resolutions. Conclusions are given in the final section.

\section{Numerical details}

\subsection{The Momentum Equations}

The Navier-Stokes equations with an added turbulent/SGS viscosity read

$$
\begin{aligned}
\frac{\partial \bar{u}_{i}}{\partial t}+\frac{\partial}{\partial x_{j}}\left(\bar{u}_{i} \bar{u}_{j}\right) & =\delta_{1 i}-\frac{1}{\rho} \frac{\partial \bar{p}}{\partial x_{i}}+\frac{\partial}{\partial x_{j}}\left[\left(\nu+\nu_{T}\right) \frac{\partial \bar{u}_{i}}{\partial x_{j}}\right] \\
\frac{\partial \bar{u}_{i}}{\partial x_{i}} & =0
\end{aligned}
$$

where $\nu_{T}=\nu_{t}\left(\nu_{t}\right.$ denotes the turbulent RANS viscosity) close to the wall for $y \leq y_{m l}$, otherwise $\nu_{T}=\nu_{S G S}$. The location at which the switch is made from URANS to LES is called the interface and is located at $y_{m l}$ from each 
wall

URANS region

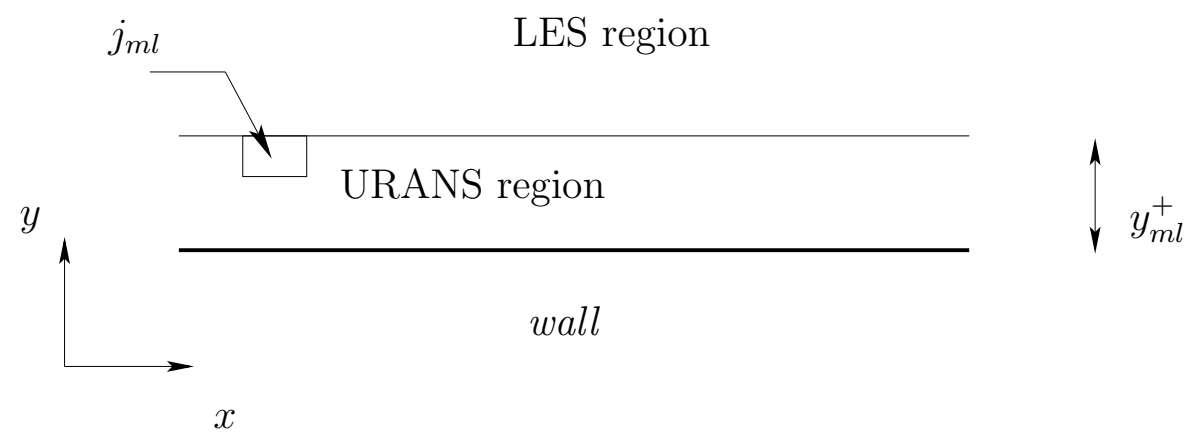

Figure 1: The LES and URANS regions. The interface is located at $y^{+}=y_{m l}^{+}$and the number of cells in the URANS region in the wall-normal direction is $j_{m l}$.

wall, see Fig. 1. The turbulent viscosity, $\nu_{T}$, is computed from an algebraic turbulent length scale, see Table 1 , and a transport equation is solved for $k_{T}$, see below. The density is set to one in all simulations. A driving constant pressure gradient, $\delta_{1 i}$, is included in the streamwise momentum equation.

\subsection{Hybrid LES-RANS}

A one-equation model is employed in both the URANS region and the LES region and reads

$$
\begin{aligned}
\frac{\partial k_{T}}{\partial t}+\frac{\partial}{\partial x_{j}}\left(\bar{u}_{j} k_{T}\right) & =\frac{\partial}{\partial x_{j}}\left[\left(\nu+\nu_{T}\right) \frac{\partial k_{T}}{\partial x_{j}}\right]+P_{k_{T}}-C_{\varepsilon} \frac{k_{T}^{3 / 2}}{\ell} \\
P_{k_{T}} & =-\tau_{i j} \bar{s}_{i j}, \quad \tau_{i j}=-2 \nu_{T} \bar{s}_{i j}
\end{aligned}
$$


where $\nu_{T}=c k^{1 / 2} \ell$. In the inner region $\left(y \leq y_{m l}\right) k_{T}$ corresponds to RANS turbulent kinetic energy, $k$; in the outer region $\left(y>y_{m l}\right)$ it corresponds to subgrid-scale kinetic turbulent energy, $k_{S G S}$. The coefficients are different in the two regions, see Table 1 . No special treatment is applied in the equations at the matching plane except that the form of the turbulent viscosity and the turbulent length scale are different in the two regions.

\subsection{The Numerical Method}

An incompressible, finite volume code is used (Davidson and Peng, 2003). For space discretization, central differencing is used for all terms except the convection term in the $k_{T}$ equation, for which the hybrid central/upwind scheme is employed. The Crank-Nicolson scheme is used for time discretization of all equations. The numerical procedure is based on an implicit, fractional step technique with a multigrid pressure Poisson solver (Emvin, 1997)

\begin{tabular}{|c|c|c|}
\hline & URANS region & LES region \\
\hline$\ell$ & $\kappa c_{\mu}^{-3 / 4} n\left[1-\exp \left(-0.2 k^{1 / 2} n / \nu\right)\right]$ & $\ell=\Delta$ \\
$\nu_{T}$ & $\kappa c_{\mu}^{1 / 4} k^{1 / 2} n\left[1-\exp \left(-0.014 k^{1 / 2} n / \nu\right)\right]$ & $0.07 k^{1 / 2} \ell$ \\
$C_{\varepsilon}$ & 1.0 & 1.05 \\
\hline
\end{tabular}

Table 1: Turbulent viscosities and turbulent length scales in the URANS and LES regions. $n$ denotes the distance to the nearest wall. $\Delta=(\delta V)^{1 / 3}$ 


\begin{tabular}{|c|c|c|c|c|c|c|c|c|}
\hline Case & $\boldsymbol{\Delta} \boldsymbol{x}$ & $\boldsymbol{\Delta} \boldsymbol{z}$ & $\boldsymbol{N}_{\boldsymbol{x}}$ & $\boldsymbol{N}_{\boldsymbol{z}}$ & $\boldsymbol{\Delta} \boldsymbol{x}^{+}$ & $\boldsymbol{\Delta} \boldsymbol{z}^{+}$ & $\boldsymbol{\delta} / \boldsymbol{\Delta} \boldsymbol{x}$ & $\boldsymbol{\delta} / \boldsymbol{\Delta} \boldsymbol{z}$ \\
\hline Baseline & 0.1 & 0.05 & 64 & 64 & 400 & 200 & 10 & 20 \\
$\mathbf{0 . 5} \boldsymbol{\Delta} \boldsymbol{x}$ & 0.05 & 0.05 & 128 & 64 & 200 & 200 & 20 & 20 \\
$\mathbf{0 . 5} \boldsymbol{\Delta} \boldsymbol{z}$ & 0.1 & 0.025 & 64 & 128 & 400 & 100 & 10 & 40 \\
$\mathbf{2} \boldsymbol{\Delta} \boldsymbol{x}$ & 0.2 & 0.05 & 32 & 64 & 800 & 200 & 5 & 20 \\
$\mathbf{2} \boldsymbol{\Delta} \boldsymbol{z}$ & 0.1 & 0.1 & 64 & 32 & 400 & 400 & 10 & 10 \\
\hline
\end{tabular}

Table 2: Test cases.

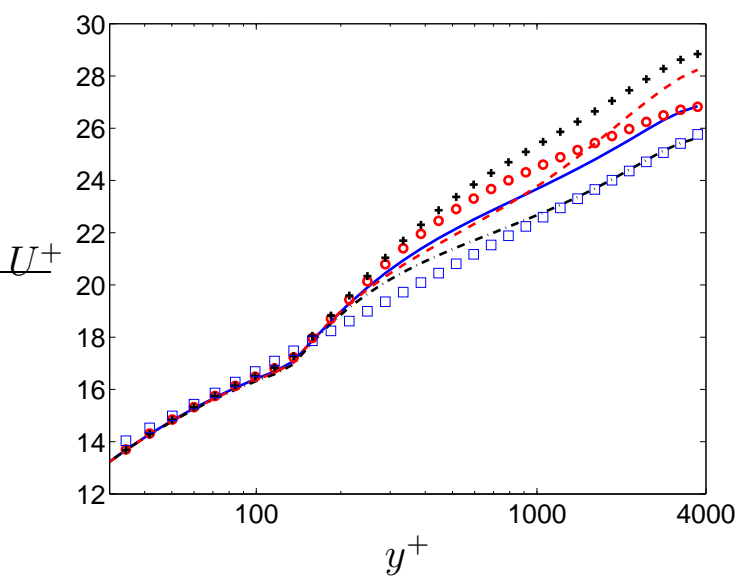

Figure 2: $U$ velocities. —— : Baseline; - - : $0.5 \Delta x ;-.-: 0.5 \Delta z ; \circ: 2 \Delta x ;+: 2 \Delta z ; \square$ : $U^{+}=\left(\ln y^{+}\right) / 0.4+5.2$.

and a non-staggered grid arrangement.

\section{Results}

The flow was computed for Reynolds number $R e_{\tau}=u_{\tau} \delta / \nu=4000(\delta$ denotes the half width of the channel), and five different computational grids 


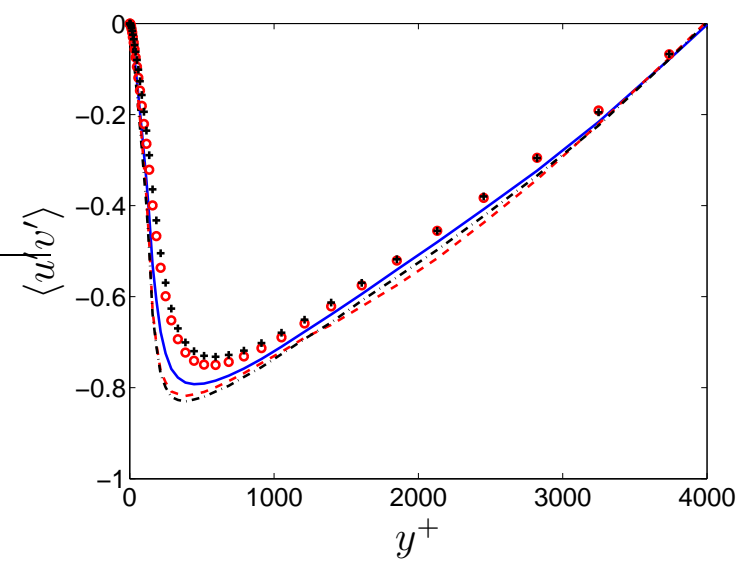

Figure 3: Resolved shear stress. For legend, see caption in Fig. 2.

are used, see Table 2. The number of cells in the $y$ direction is 80 with a constant geometric stretching of $15 \%$. This gives a smallest and largest cell height of $\Delta y_{\min }^{+}=2.2$ and $\Delta y_{\max }^{+}=520$, respectively. The grid spacing in the wall-parallel plane in viscous units is $\left(\Delta x^{+}, \Delta z^{+}\right)=(200-800,100-400)$. The extent of the domain in the $x$ and $z$ directions is 6.4 and 3.2, respectively, for all cases. The matching line is chosen along a fixed grid line at $y^{+}=125$ $(y=0.0313)$ so that 16 cells are located in the URANS region at each wall. It should be mentioned that in the present work the matching line between the URANS and the LES regions is located rather close to the wall. If the matching line were located as in $\operatorname{DES}$ (i.e. $\min \left(0.65 \Delta_{m}, y\right)$, $\left.\Delta_{m}=\max (\Delta x, \Delta y, \Delta z)\right)$, the agreement would have been much better for all five cases. However, the object of this work was not to obtain the best possible results, but to compared different ways of estimating the resolution. 
The velocity profiles are shown in Fig. 2. It can be seen that the Baseline case over-predicts the velocity in the LES region. When the grid is refined in the spanwise direction (Case $0.5 \Delta z$ ) much better agreement with the log-law is obtained. When the grid is coarsened in either the streamwise (Case $2 \Delta x$ ) or the spanwise direction (Case $2 \Delta z$ ), the agreement with the log-law deteriorates as expected. It is somewhat surprising that a grid refinement in the streamwise direction (Case $0.5 \Delta x$ ) gives worse agreement than the Baseline case. It turns out that the smaller SGS length scale is the main reason for this; if the SGS length for Case $0.5 \Delta x$ is set equal to that in the Baseline case, the velocity in the outer region decreases compared to the original Case $0.5 \Delta x$ (not shown). A grid with $(0.5 \Delta x, 0.5 \Delta z)$ gives even better agreement with the log law (not shown). We argue that the large discrepancy in the outer region for Case $0.5 \Delta x$ is a turbulence model issue. When going from the Baseline case to Case $0.5 \Delta x$, the SGS length scale is reduced by a factor of $(1 / 2)^{1 / 3}$ and the SGS shear stress will as a consequence also decrease. Since the total shear shear stress must obey the relation $y-1$ (dictated by the time-averaged streamwise momentum equation), the decrease in SGS shear stress should be compensated by a corresponding increase in the resolved shear stress, $\left|\left\langle u^{\prime} v^{\prime}\right\rangle\right|$. The increase of the magnitude of the resolved shear stress is facilitated by lower SGS dissipation because of smaller SGS viscosity. The resolved shear stress, $\left|\left\langle u^{\prime} v^{\prime}\right\rangle\right|$, does indeed increase in the inner 
region when going from the Baseline case to Case $0.5 \Delta x$, see Fig. 3. However, in the outer region - in which the velocity gradient is much smaller the flow is initially not able to increase the magnitude of the resolved shear stress. Hence, in order to satisfy the linear law of the total shear, the magnitude of the SGS shear stress must increase and the flow accomplishes this by increasing the velocity gradient for $y^{+}>1000$, see Fig. 2. Careful inspection of Fig. 3 reveals that also the magnitude of the resolved shear stress for Case $0.5 \Delta x$ is slightly larger (for $1500<y^{+}<3000$ ) than for the Baseline case. The question then arises why the agreement of the velocity profile with the log law does not deteriorate when the grid is refined in the spanwise direction (Case $0.5 \Delta z$ ). The answer is that the refinement in the spanwise direction allows additional turbulence to be resolved (the peak of $\left|\left\langle u^{\prime} v^{\prime}\right\rangle\right|$ is larger for Case $0.5 \Delta z$ than for Case $0.5 \Delta x$ ) which compensates for the decrease in the SGS shear stress. The discussion above indicates that it could be interesting to develop new SGS models in which the SGS length scale is sensitized to the magnitude of the resolved strain, $\left(2 \bar{s}_{i j} \bar{s}_{i j}\right)^{1 / 2}$; if the resolved strain is large, the length scale can be taken as $\Delta$, but if it is small the length scale should maybe be larger.

Figures 4 and 5 present the streamwise and wall-normal resolved fluctuations, respectively. As can be seen, $u_{r m s}^{2}$ and $v_{r m s}^{2}$ increases and decreases, respectively, as the grid is coarsened. The time-averaged streamwise mo- 


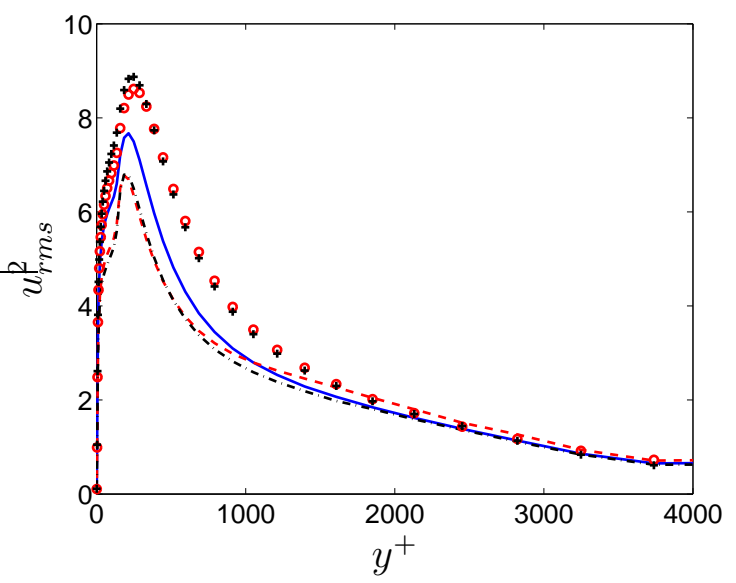

Figure 4: Resolved fluctuations in the streamwise direction. For legend, see caption in Fig. 2.

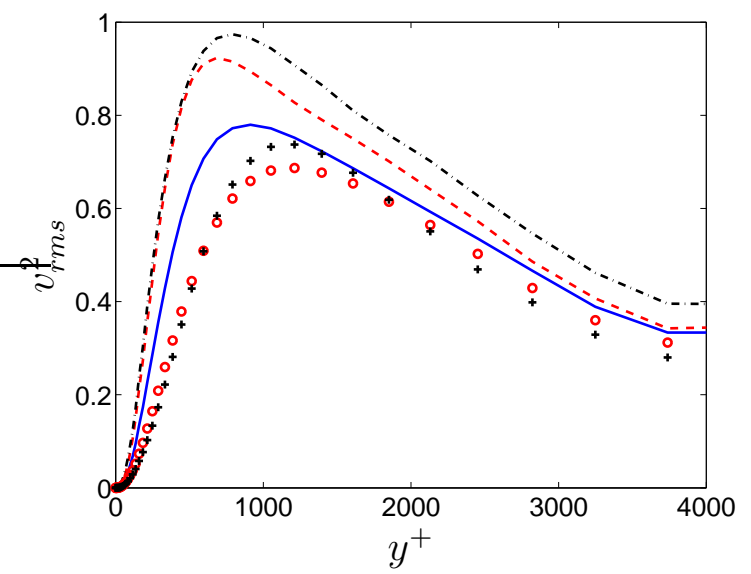

Figure 5: Resolved fluctuations in the wall-normal direction. For legend, see caption in Fig. 2.

mentum equation requires that the total shear stress must satisfy the relation $y-1$, see Fig. 3. When the grid is coarsened, the resolved part of the total shear stress decreases for $y^{+} \lesssim 1000$, mainly because the turbu- 


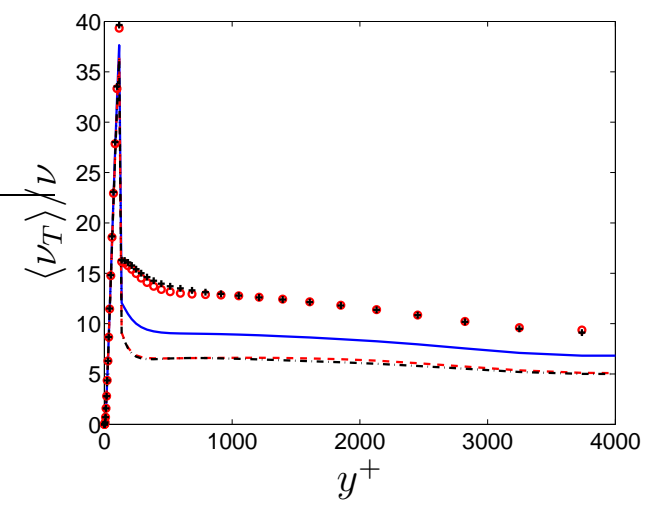

Figure 6: Turbulent viscosity. For legend, see caption in Fig. 2.

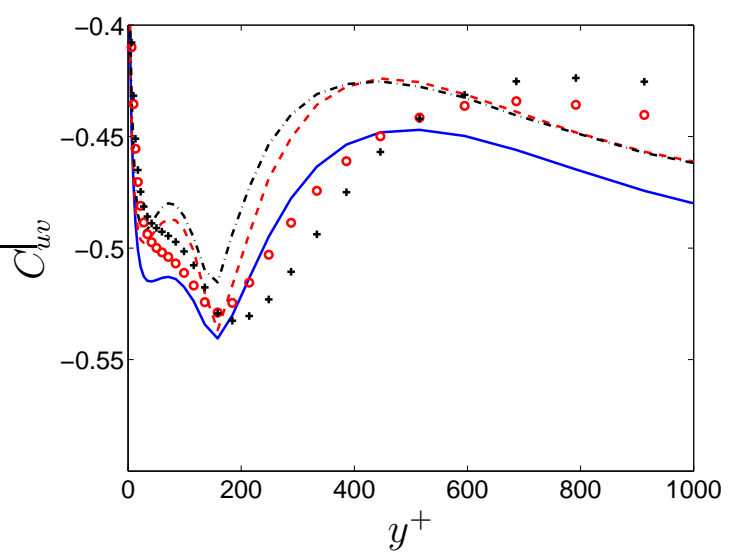

Figure 7: The correlation coefficient, $C_{u v}=\left\langle u^{\prime} v^{\prime}\right\rangle /\left(u_{r m s} v_{r m s}\right)$. For legend, see caption in Fig. 2.

lent viscosity increases with grid coarsening, see Fig. 6. Furthermore, the non-linear interaction is weakened so that, in the region of maximum $\left|\left\langle u^{\prime} v^{\prime}\right\rangle\right|$ (i.e. $200<y^{+}<600$ ), the correlation between the streamwise and wallnormal resolved fluctuations is diminished, see Fig. 7 (this is also seen in coarse DNS (Davidson and Billson, 2006)). Initially, this results in too small 


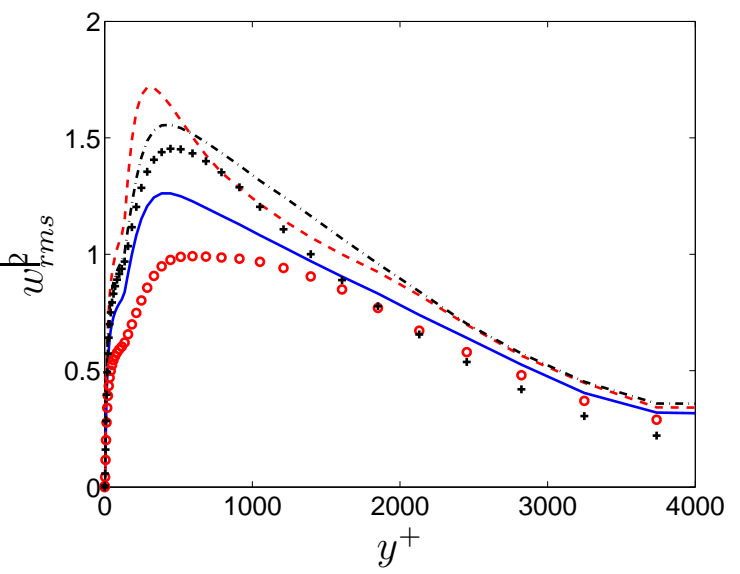

Figure 8: Resolved fluctuations in the spanwise direction. For legend, see caption in Fig. 2.
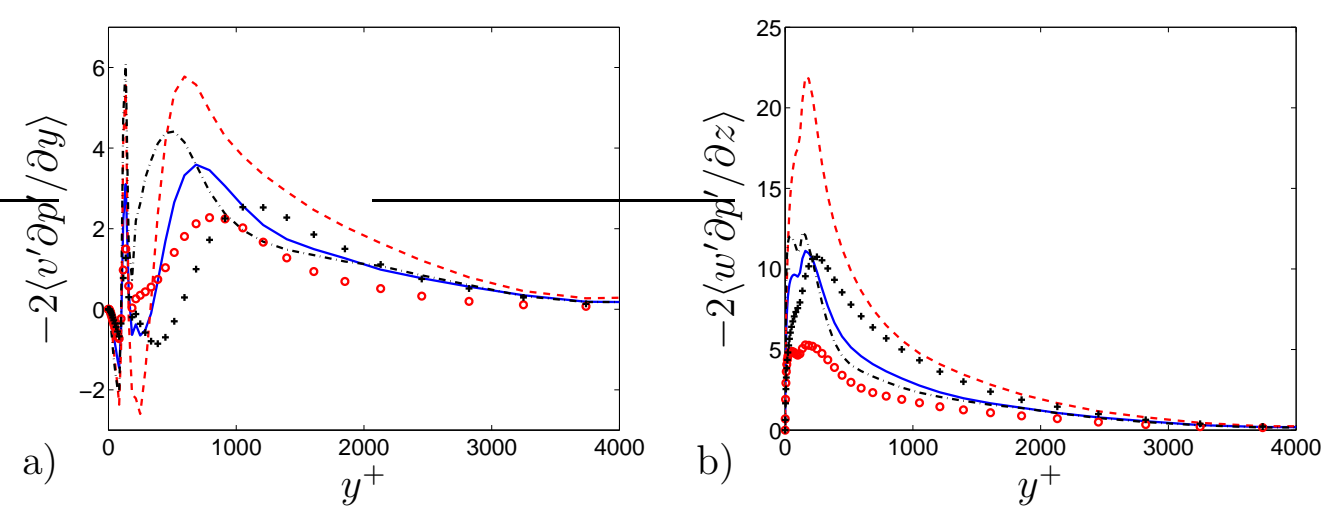

Figure 9: Pressure-gradient-velocity terms in the (a) $v_{r m s}^{2}$ and (b) $w_{r m s}^{2}$ equations. For legend, see caption in Fig. 2.

a resolved shear stress. The equations respond by increasing the resolved fluctuations by increasing the bulk velocity and hence the velocity gradient, $\partial \bar{u} / \partial y$, which is the main agent for producing resolved turbulence. The bulk velocity, and hence the resolved fluctuations, are increased until the resolved shear stress is large enough so that the total shear stress satisfies the linear 

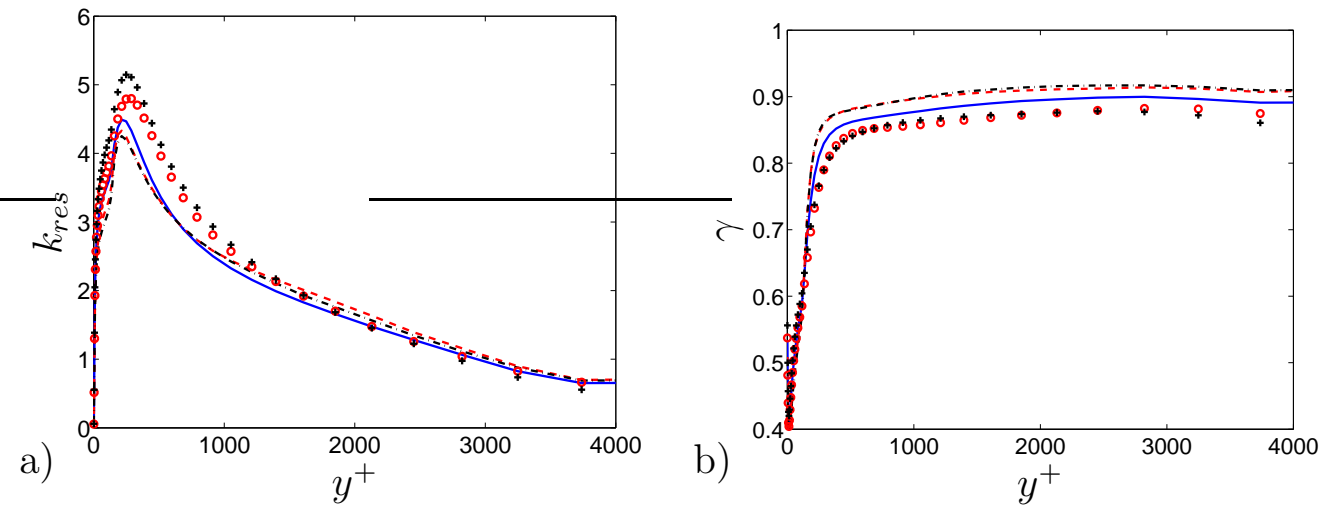

Figure 10: Resolved turbulent kinetic energy kinetic energy and the ratio $\gamma$ (Eq. 2). For legend, see caption in Fig. 2.

relation. Because of the damping effect of the wall on $v^{\prime}$, the equations increase $u^{\prime}$ until the linear relation is obtained. This explains the large values of $u_{r m s}^{2}$ in Fig. 4 for the coarse resolutions. It should be stressed that the process of increasing the resolved fluctuations until the total shear stress satisfies the linear relation $y-1$ does not occur in a general flow case with an inlet and outlet (i.e. a flow case with no prescribed driving pressure gradient). In a general flow case, the magnitude of the resolved shear stresses will simply remain too small.

The spanwise fluctuations increase when the grid is coarsened in the spanwise direction, whereas they decrease upon coarsening in the streamwise direction, see Fig. 8. They increase when the grid is refined. Furthermore, the location of the peak is not affected neither by grid coarsening nor grid refinement. This behaviour is completely different from that of the wall- 
normal stresses. The different behavior of the wall-normal fluctuations and the spanwise fluctuations is explained by Fig. 9, where the pressure-gradientvelocity terms are presented; these terms are the main source terms in the transport equations of $v_{r m s}^{2}$ and $w_{r m s}^{2}$. It can be seen that the term in the $v_{r m s}^{2}$ equation - like $v_{r m s}^{2}$ itself - decreases/increases in the LES region for $y^{+}<1000$ when the grid is coarsened/refined and that the peak of the source term moves away from the wall. The term in the $w_{r m s}^{2}$ equation, however, shows a different behavior when the grid is coarsened in the spanwise or the streamwise direction: it increases in the former case whereas it increases in the latter case. When the grid is refined in the streamwise direction, the source term increases. This agrees with the behavior of $w_{r m s}^{2}$, see Fig. 8. A conclusion from the discussion above is that it is crucial to properly resolve the interaction between fluctuating resolved velocities and pressure.

The resolved turbulent kinetic energy is presented in Fig. 10a, and since it is dominated by the streamwise fluctuations, its behavior is similar to that of $u_{r m s}^{2}$. Pope (2004) suggests that when $80 \%$ of the turbulent kinetic energy is resolved, i.e.

$$
\gamma=\frac{k_{r e s}}{<k_{T}>+k_{r e s}}>0.8, \quad k_{r e s}=\frac{1}{2}\left\langle u_{i}^{\prime} u_{i}^{\prime}\right\rangle
$$

the LES can be consider to be well-resolved. Figure 10b presents this ratio and as can be seen it is, in the LES region, larger than $85 \%$ for all grids. Hence this quantity does not seem to be a good measure of the resolution. 


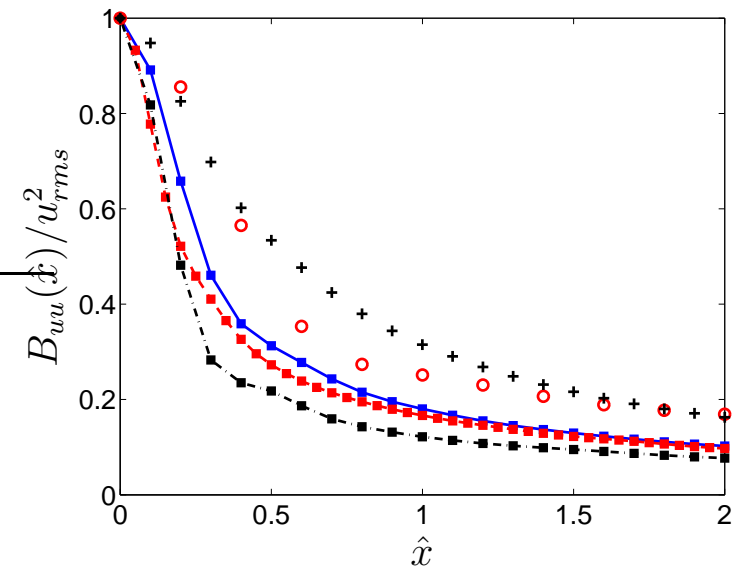

Figure 11: Normalized two-point correlation $B_{u u}(\hat{x}) / u_{r m s}^{2} \cdot y=0.11$. For legend, see caption in Fig. 2. Markers on the lines show the resolution.

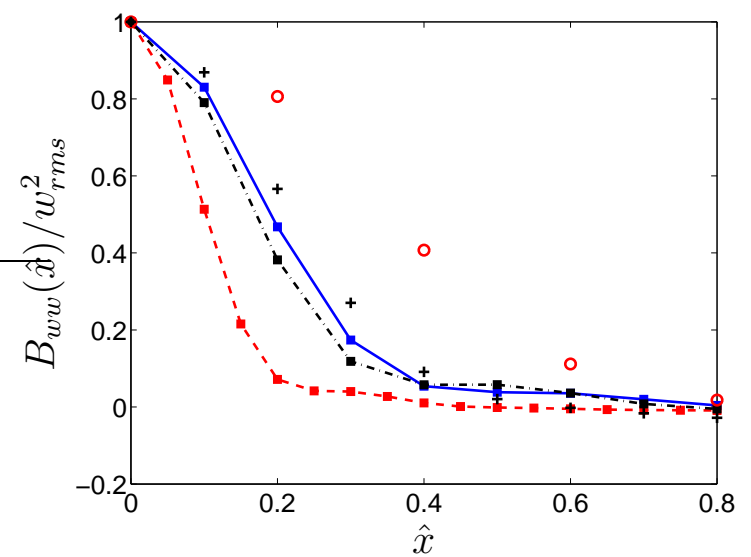

Figure 12: Normalized two-point correlation $B_{w w}(\hat{x}) / w_{r m s}^{2} . y=0.11$. For legend, see caption in Fig. 2. Markers on the lines show the resolution.

Figures 11, 12 and 13 present the streamwise and spanwise two-point correlations, which are defined as $B_{u u}(\hat{x})=\left\langle u^{\prime}(x) u^{\prime}(x-\hat{x})\right\rangle, B_{w w}(\hat{x})=$ $\left\langle w^{\prime}(x) w^{\prime}(x-\hat{x})\right\rangle$ and $B_{w w}(\hat{z})=\left\langle w^{\prime}(z) w^{\prime}(z-\hat{z})\right\rangle$, respectively. The two-point 


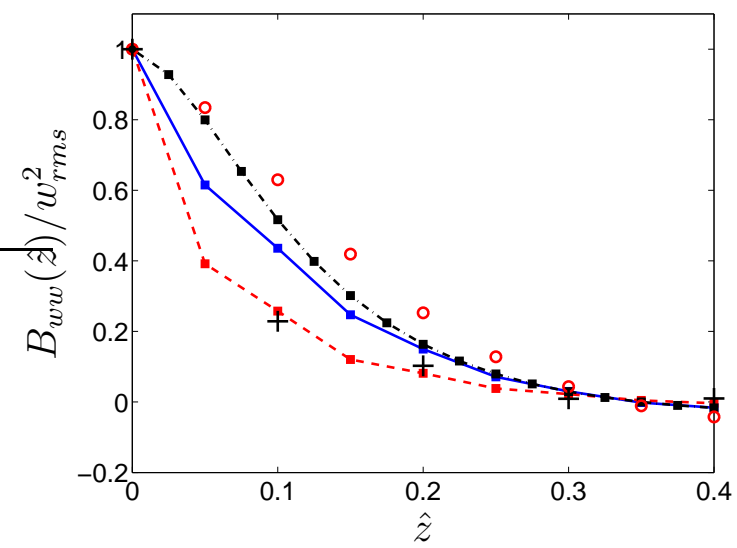

Figure 13: Normalized two-point correlation $B_{w w}(\hat{z}) / w_{r m s}^{2} . y=0.11$. Markers on the lines show the resolution. For legend, see caption in Fig. 2.

correlations are all presented for $y^{+} \simeq 440(y=0.11)$; they do not vary much across the channel. The two-point correlations are normalized with $u_{r m s}^{2}$ and $w_{r m s}^{2}$, respectively. In Fig. 11, 12, the two-point correlations increase when the grid is coarsened (Cases $2 \Delta x$ and $2 \Delta z$ ) because the smaller scales are not resolved; for these coarse resolutions the streamwise two-point correlations are dominated by the "superstreaks" (Piomelli et al., 2003; Keating and Piomelli, 2006). When the grid is refined (Cases $0.5 \Delta x$ and $0.5 \Delta z$ ), the two-point correlations decrease, as expected. For Case $2 \Delta x$, the two-point correlation formed with $u^{\prime}$ (Fig. 11) falls down to 0.4 within four cells. In the spanwise direction it is even worse: for Case $2 \Delta z$ the two-point correlation goes to zero within two cells (Fig. 13). The reason is that the grid is so coarse that the non-linear process of generating turbulence cannot be 


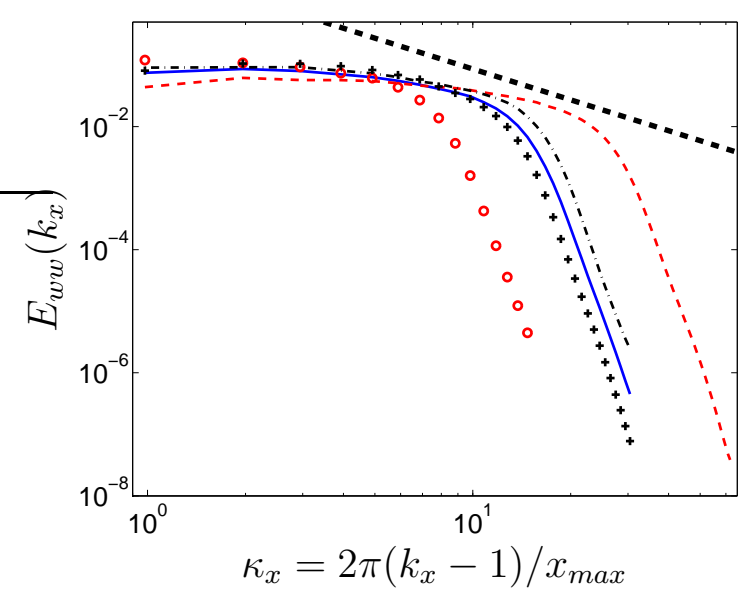

Figure 14: Energy spectra $E_{w w}\left(k_{x}\right) . y=0.11$. The thick dashed line shows -1 slope. For legend, see caption in Fig. 2.

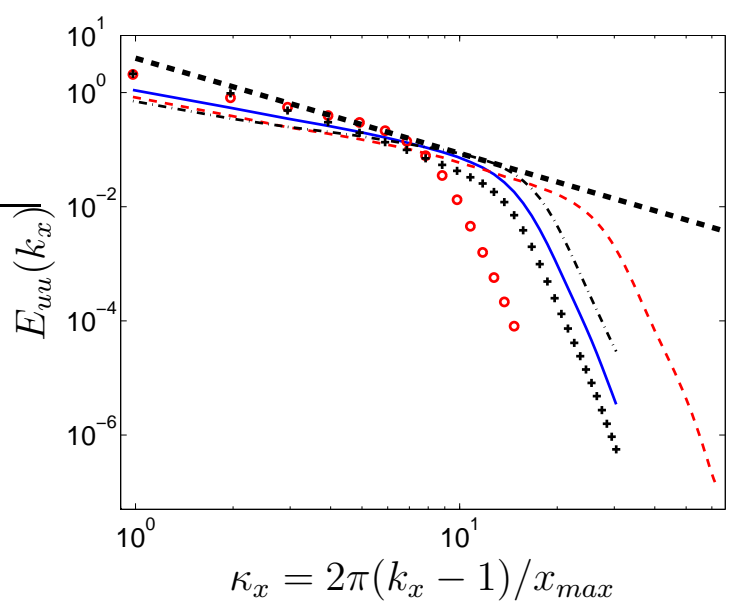

Figure 15: Energy spectra $E_{u u}\left(k_{x}\right) \cdot y=0.11$. The thick dashed line shows -1 slope. For legend, see caption in Fig. 2.

sustained. This is a clear indication that the resolution is too poor for Cases $2 \Delta x$ and $2 \Delta z$.

Figures 14 and 15 present the one-dimensional energy spectra $E_{w w}\left(k_{x}\right)$ 


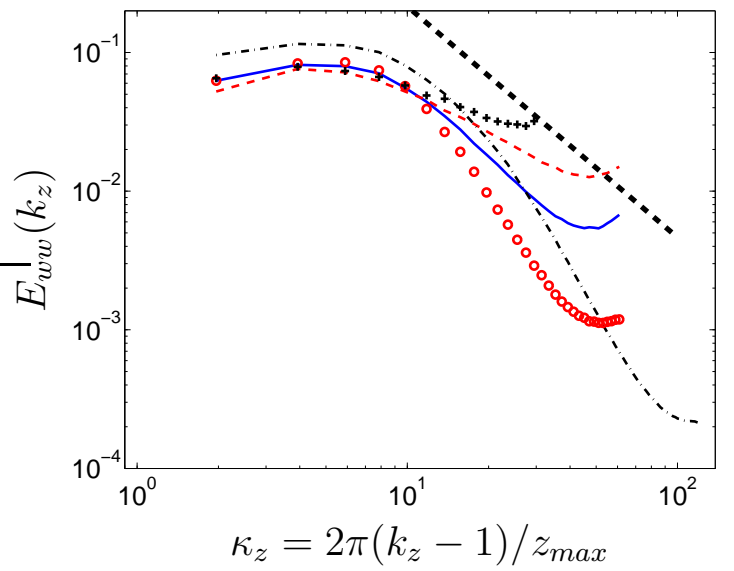

Figure 16: Energy spectra $E_{w w}\left(k_{z}\right) . y=0.11$. The thick dashed line shows $-5 / 3$ slope. For legend, see caption in Fig. 2.

and $E_{u u}\left(k_{x}\right)$. The smallest wavenumber is $\kappa_{x, \min }=2 \pi / x_{\max }=2 \pi / 6.4=$ 0.98. The largest wavenumber included in the plot is $\kappa_{x, \max }=2 \pi / 2 \Delta x$, where we have assumed that two cells are required to resolve a wavelength. For the Baseline case, for example, this gives $\kappa_{x, \max }=\pi / 0.1=31$. The energy spectrum $E_{w w}\left(k_{x}\right)=\hat{W}^{2}$, for example, is computed by the DFT (Discrete Fourier Transform) of $w^{\prime}$

$$
\begin{aligned}
\hat{W}\left(k_{x}\right) & =\frac{1}{N_{x}} \sum_{n=1}^{N_{x}} w^{\prime}(n)\left[\cos \left(\frac{2 \pi(n-1)\left(k_{x}-1\right)}{N_{x}}\right)\right. \\
& \left.-\imath \sin \left(\frac{2 \pi(n-1)\left(k_{x}-1\right)}{N_{x}}\right)\right]
\end{aligned}
$$

where $\hat{W}$ are the complex Fourier coefficients of $w^{\prime}$ and $\imath^{2}=-1$; the normalized streamwise coordinate appears at the right side as $(n-1) / N_{x}=x / x_{\max }$. The $w_{r m s}^{2}$ can be retrieved by summing the square of the real and imaginary 
parts of $\hat{W}$, i.e.

$$
w_{r m s}^{2}=\sum_{n=1}^{N_{x}}\left(\operatorname{Re}\left(\hat{W}\left(k_{x}\right)\right)\right)^{2}+\left(\operatorname{Im}\left(\hat{W}\left(k_{x}\right)\right)\right)^{2}
$$

The energy spectra are all presented for $y^{+} \simeq 440(y=0.11)$ but, when plotted in log-log as in Figs. 14 and 15, they show the same behavior across the channel, the main difference being that they are shifted along the $E_{w w}$ $E_{u u}$ axes as $w_{r m s}^{2}$ and $u_{r m s}^{2}$ vary across the channel. For comparison, a slope of " -1 " is included in the figures. For high wavenumbers $\left(k_{x}>7\right.$ for $E_{w w}$ and $k_{x}>10$ for $E_{u u}$ ), the streamwise energy spectra for the Case $2 \Delta x$ exhibits a clear "dissipation" range (i.e. a steeper slope than $-5 / 3$ ). For the other cases the dissipation range starts at higher wavenumbers. If the energy spectra had been obtained from DNS or experiments, it would indeed have been a dissipation range but, in the present case, we are using a turbulence model and hence what is seen in the energy spectra is an $S G S$ dissipation range.

Figure 16 shows the spanwise energy spectra of the spanwise fluctuations. A line with a $-5 / 3$ slope is included for comparison. As can be seen, no $-5 / 3$ range exists. The energy spectra for all cases show a tendency of pile-up of energy in the small scales. This phenomenon is present across the channel.

The spanwise energy spectra presented, Fig. 16, do not actually give much guidance as to whether the resolution is sufficient. With an effort, one could find a $-5 / 3$ range for the Baseline case, Cases $0.5 \Delta x$ and $0.5 \Delta z$, indicating 

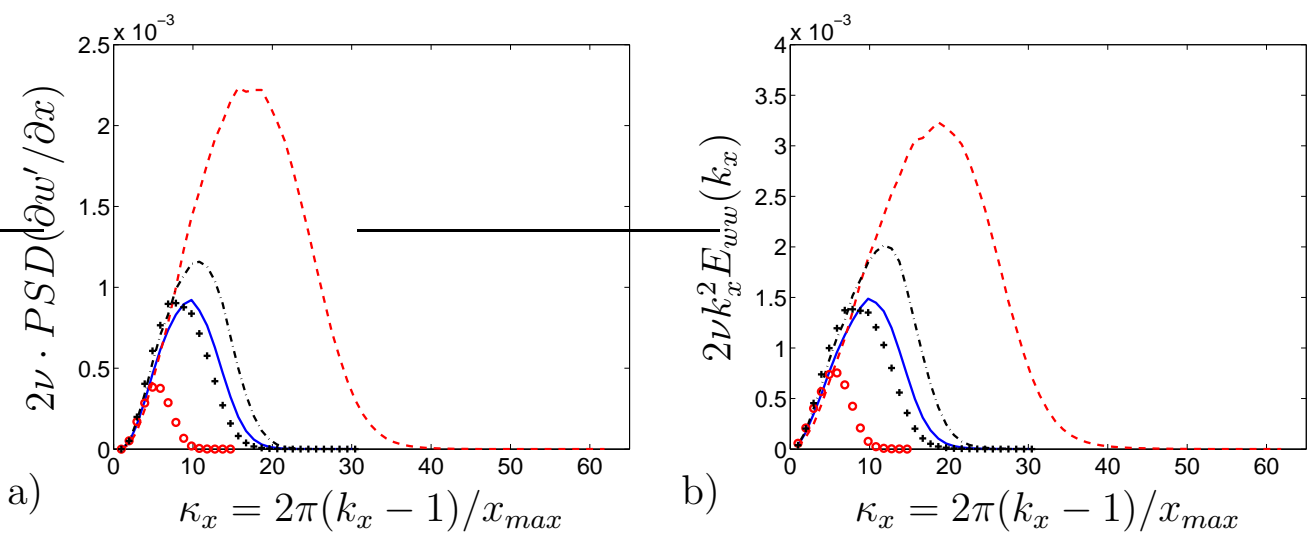

Figure 17: Energy spectra of an exact and approximated spanwise component of viscous dissipation versus streamwise wavenumber. $y=0.11$. For legend, see caption in Fig. 2 .

that these resolutions all are sufficient. But from the two-point correlations in Fig. 13 we find that the largest scales are covered by 4,4 and 8 cells, respectively. Only Case $0.5 \Delta z$ could be considered to be sufficient. The streamwise energy spectra, Figs. 14 and 15, indicate that the resolution is too coarse for all cases (no $-5 / 3$ range). The two-point correlation for Case $0.5 \Delta x$ (Fig. 11), for example, shows that the largest scales are covered by some 16 cells which should be sufficient.

The energy spectra in Fig. 16 indicate that SGS dissipation is active at relatively low wave numbers (the pile-up of energy at high wavenumbers shows that the SGS dissipation is small at high wavenumbers). To investigate this further, we will study the spectra of the dissipation. The two-point correlation, $B_{w w}(\hat{z})$, is the inverse DFT of the symmetric energy spectrum, 

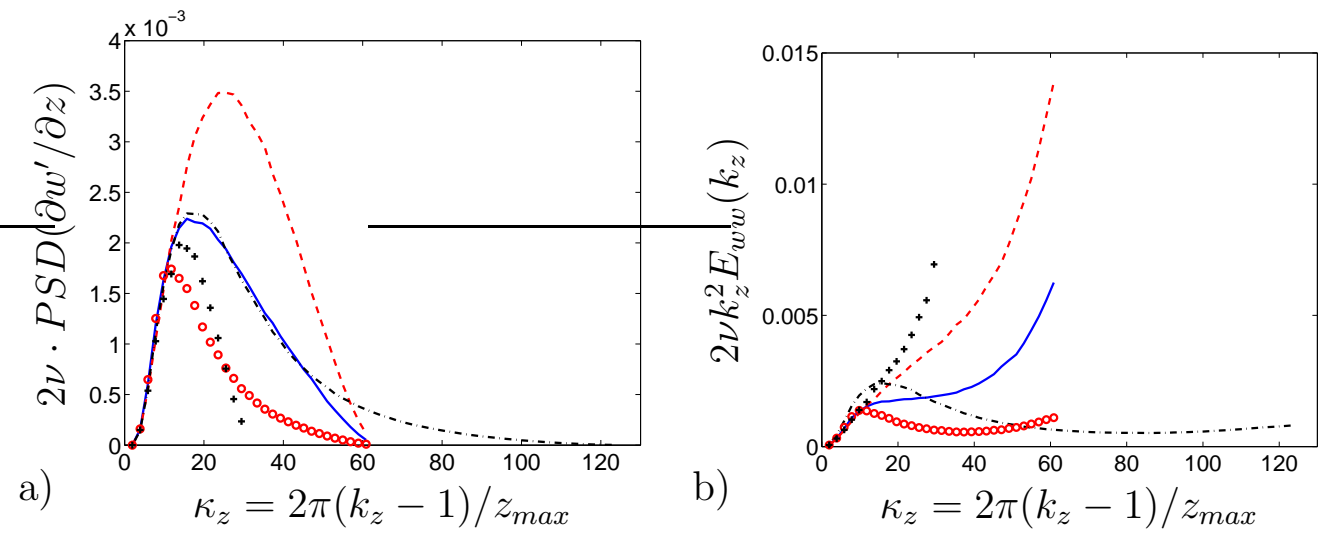

Figure 18: Energy spectra of an exact and approximated spanwise component of viscous dissipation versus spanwise wavenumber. $y=0.11$. For legend, see caption in Fig. 2 .

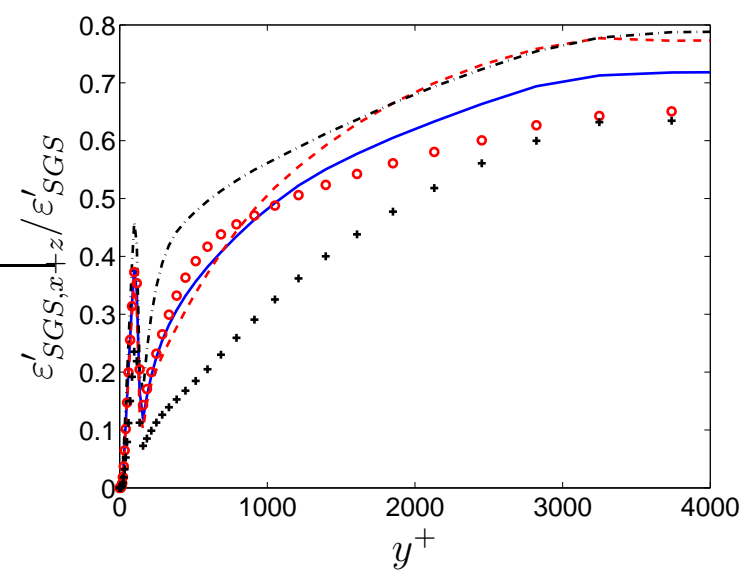

Figure 19: Ratio of SGS dissipation due to resolved fluctuations in the wall-parallel plane to the total one, see Eq. 9. For legend, see caption in Fig. 2.

$E_{w w}$, i.e.

$$
B_{w w}\left(\hat{z} / z_{\max }\right)=\sum_{k_{z}=1}^{N_{z}} E_{w w}\left(k_{z}\right) \cos \left(\frac{2 \pi\left(k_{z}-1\right)(n-1)}{N_{z}}\right)
$$

where $N_{z}$ is the number of cells in the spanwise direction; the normalized 


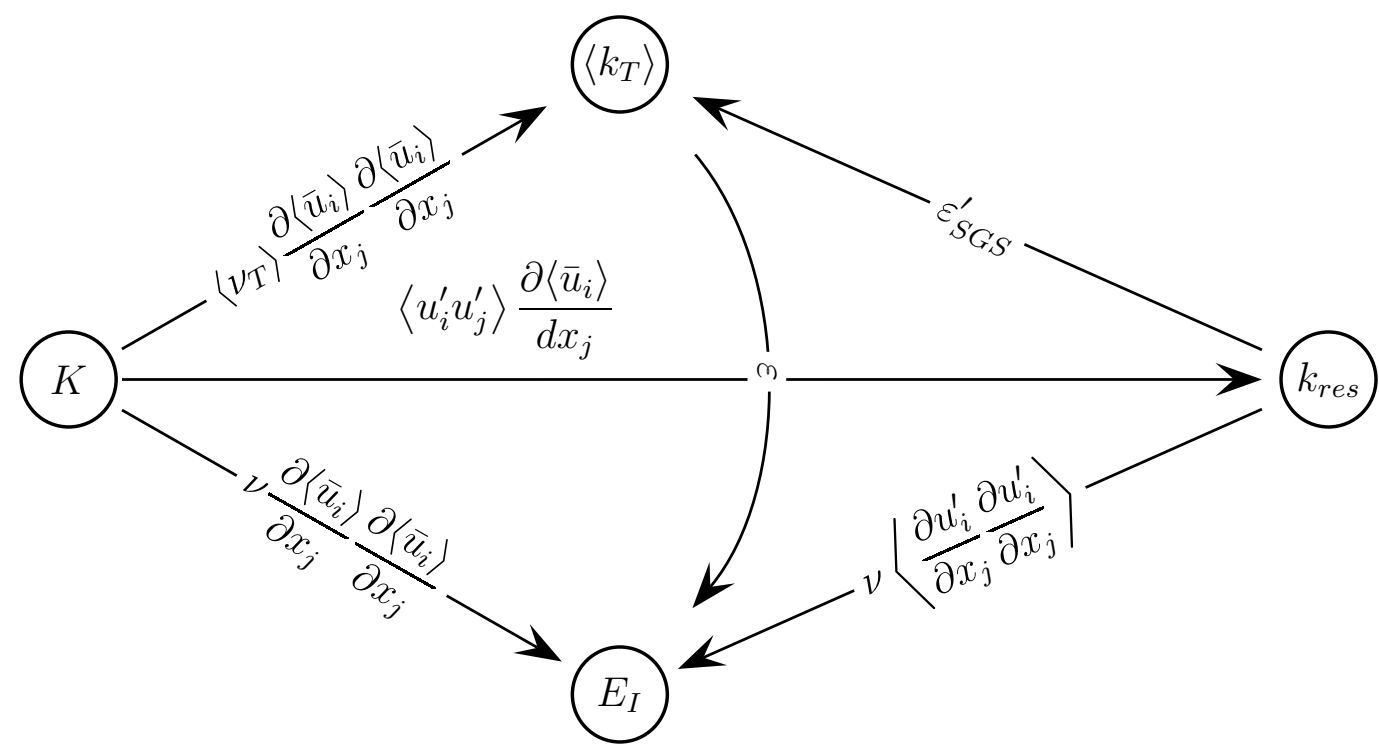

Figure 20: Transfer of kinetic turbulent energy between time-averaged, resolved, modelled kinetic energy and internal energy. $K=\frac{1}{2}\left\langle\bar{u}_{i}\right\rangle\left\langle\bar{u}_{i}\right\rangle$ and $k_{\text {res }}=\frac{1}{2}\left\langle u_{i}^{\prime} u_{i}^{\prime}\right\rangle$ denote timeaveraged kinetic and resolved turbulent kinetic energy, respectively. $E_{I}$ denotes internal energy. $\varepsilon$ is viscous dissipation at subgrid scales, see Eq. 10.
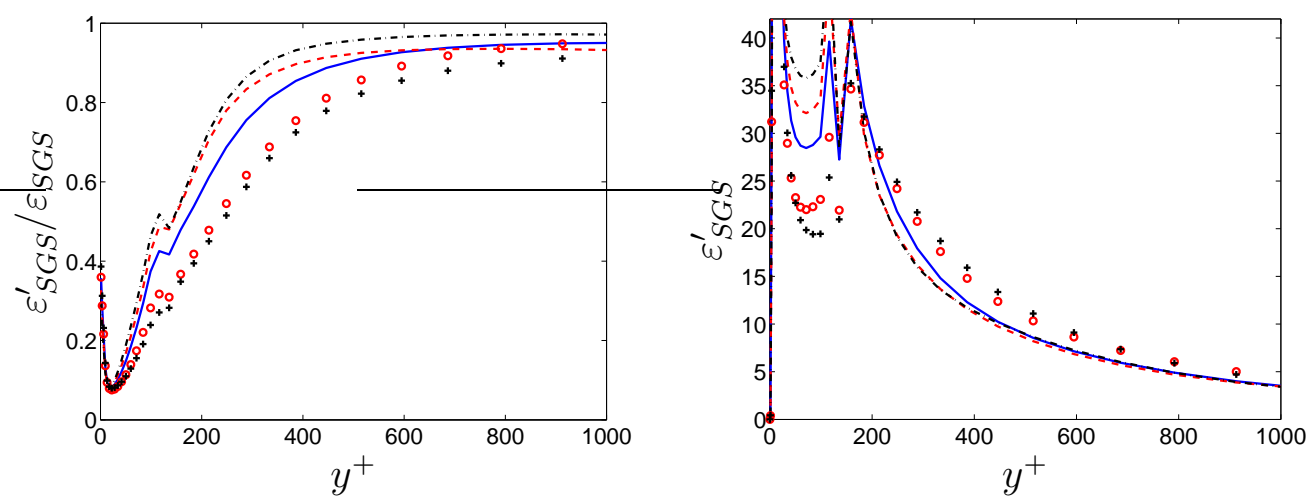

Figure 21: SGS dissipation due to resolved fluctuations, $\varepsilon_{S G S}^{\prime}$, see Eq. 9. For legend, see caption in Fig. 2. 
spanwise separation distance appears at the right side as $(n-1) / N_{z}=\hat{z} / z_{\max }$. $\varepsilon_{w z}$ can — in theory — be obtained from (Hinze, 1975)

$$
\varepsilon_{w z}=2 \nu\left\langle\left(\frac{\partial w^{\prime}}{\partial z}\right)^{2}\right\rangle=\left.2 \nu \frac{\partial^{2} B_{w w}(\hat{z})}{\partial \hat{z}^{2}}\right|_{\hat{z}=0}=2 \nu \sum_{k_{z}=1}^{N_{z}} \kappa_{z}^{2} E_{w w}\left(k_{z}\right)
$$

where $\kappa_{z}=2 \pi\left(k_{z}-1\right) / z_{\max }$. However, this relation is not satisfied at the discrete level, because the derivative $\partial w^{\prime} / \partial z$ cannot be evaluated exactly in a finite-volume approach (the relation would have been satisfied had we used a spectral method solving for the Fourier coefficients), and thus

$$
\varepsilon_{w z, F V} \neq 2 \nu \sum_{k_{z}=1}^{N_{z}} \kappa_{z}^{2} E_{w w, F V}\left(k_{z}\right)
$$

where subscript $F V$ denotes Finite Volume. Instead, a DFT of $\partial w^{\prime} / \partial z$ as

$$
\begin{aligned}
\hat{D}_{z}\left(k_{z}\right) & =\frac{1}{N_{z}} \sum_{n=1}^{N_{z}} \frac{\partial w^{\prime}(n)}{\partial z}\left[\cos \left(\frac{2 \pi(n-1)\left(k_{z}-1\right)}{N_{z}}\right)\right. \\
& \left.-\imath \sin \left(\frac{2 \pi(n-1)\left(k_{z}-1\right)}{N_{z}}\right)\right]
\end{aligned}
$$

must be formed where $\hat{D}_{z}$ are the complex Fourier coefficients of $\partial w^{\prime} / \partial z$. Then the Power Spectral Density, i.e. $\hat{D}_{z} * \hat{D}_{z}^{*}$, where superscript $*$ denotes complex conjugate, can be formed. Now indeed (cf. Eq. 4)

$$
\varepsilon_{w z, F V}=\varepsilon_{w z, e x a c t}=2 \nu \sum_{k_{z}=1}^{N_{z}} \hat{D}_{z} * \hat{D}_{z}^{*}=2 \nu \sum_{k_{z}=1}^{N_{z}} P S D\left(\partial w^{\prime} / \partial z\right) .
$$

Figures 17 and 18 present the energy spectra of the resolved velocity gradients $\partial w^{\prime} / \partial x$ and $\partial w^{\prime} / \partial z$. As mentioned above, $\kappa_{x}^{2} E_{w w}\left(k_{x}\right)$ and $P S D\left(\partial w^{\prime} / \partial x\right)$, and $\kappa_{z}^{2} E_{w w}\left(k_{z}\right)$ and $P S D\left(\partial w^{\prime} / \partial z\right)$, respectively, should in theory be equal; 
as can be seen, the former are larger than the latter. The discrepancy is in effect a measure of the inaccuracy of the finite volume method in estimating a derivative, and the error becomes larger for small spanwise scales (large spanwise wavenumbers, $\kappa_{z}$ ). Although Eq. 5 is formally exact, the correct way to create a spectrum of the computed results is to use Eqs. 7 and 8. The reason is that, in Eqs. 7 and 8, the velocity gradients are estimated in the same way as when predicting the flow, whereas the velocity gradients in Eq. 5 are estimated with higher accuracy (actually exactly) than when predicting the flow. Furthermore, the predicted dissipation in the physical and spectral space agrees when Eq. 7 is used, whereas they do not when Eq. 5 is used.

It is commonly assumed that the major part of the SGS dissipation takes place close to the cut-off, i.e. at high resolved wavenumbers. Figures 17a and 18a show that this is not the case at all. The peaks of the resolved velocity gradients are located at $k_{x}, k_{z}<20$. This explains the strong decay in the energy spectra in Figs. 14, 15 and 16. For wavenumbers, $k_{x}$, larger than 20, no dissipation takes place for any resolution except for Case $0.5 \Delta x$, see Fig. 17a.

The dissipation energy spectra in the spanwise direction show a large discrepancy at high wavenumbers between the exact ones (Eqs. 7, 8, Fig. 18a) and those computed from the energy spectra of $w^{\prime}$ (Eq. 5, Fig. 18b). This is an indication of how poorly the velocity gradients are resolved for the higher 
wavenumbers.

When the dissipation spectra presented above are plotted in the center of the channel, they do not change a great deal except that the peaks are shifted slightly towards lower wavenumbers.

Above, components of the energy spectra for the viscous dissipation (e.g. $\left.\nu\left(\partial w^{\prime} / \partial z\right)^{2}\right)$ rather than for the SGS dissipation (e.g. $\left.\nu_{T}\left(\partial w^{\prime} / \partial z\right)^{2}\right)$ are presented. The main reason for this is that it is only for the viscous dissipation that Eq. 5, in theory, holds. Actually, the exact spectrum of $\nu_{T}\left(\partial w^{\prime} / \partial z\right)^{2}$ has been computed (note that in this case the Fourier coefficients in Eq. 7 are formed with $\nu_{T}^{0.5} \partial w^{\prime} / \partial z$, whose physical meaning is somewhat obscure), and it is found that the two energy spectra are very similar; one difference is that, unlike for the viscous dissipation, the first Fourier mode is non-zero for the SGS dissipation because $\left\langle\nu_{T}^{0.5} \partial w^{\prime} / \partial z\right\rangle \neq 0$ whereas $\left\langle\nu \partial w^{\prime} / \partial z\right\rangle=0$.

Above we have only analyzed the dissipation spectra due to spanwise and streamwise derivatives. One reason is that the spanwise and streamwise components of the SGS dissipation make an important contribution to the total one. Figure 19 shows the ratio of the SGS dissipation in the wall-parallel plane, $\varepsilon_{S G S, x+z}^{\prime}$, to the total SGS dissipation, $\varepsilon_{S G S}^{\prime}$, where

$$
\begin{aligned}
\varepsilon_{S G S}^{\prime} & =\varepsilon_{S G S}-\varepsilon_{\langle S G S\rangle}=\left\langle\nu_{T} \frac{\partial \bar{u}_{i}}{\partial x_{j}} \frac{\partial \bar{u}_{i}}{\partial x_{j}}\right\rangle-\left\langle\nu_{T}\right\rangle\left(\frac{\partial\langle\bar{u}\rangle}{\partial y}\right)^{2} \\
\varepsilon_{S G S, x+z}^{\prime} & =\left\langle\nu_{T} \frac{\partial \bar{u}_{i}}{\partial x_{1}} \frac{\partial \bar{u}_{i}}{\partial x_{1}}+\nu_{T} \frac{\partial \bar{u}_{i}}{\partial x_{3}} \frac{\partial \bar{u}_{i}}{\partial x_{3}}\right\rangle
\end{aligned}
$$

The contribution of $\varepsilon_{S G S, x+z}^{\prime}$ to $\varepsilon_{S G S}^{\prime}$ is close to $35 \%$ or larger for all cases 
except Case $2 \Delta z$, see Fig. 19. Another reason why we have not included the wall-normal derivative in the analyze in Figs. 17 and 18 is that Eq. 5 does not hold for this derivative ( $x_{2}$ is not an homogeneous direction).

Figure 20 presents the flow of kinetic energy between the time-averaged kinetic energy, $\left\langle\bar{u}_{i}\right\rangle\left\langle\bar{u}_{i}\right\rangle / 2$, the resolved fluctuating kinetic energy, $\left\langle u_{i}^{\prime} u_{i}^{\prime}\right\rangle / 2$, the modelled (SGS) kinetic energy, $\left\langle k_{T}\right\rangle$, and the energy flow of all three kinetic energies down to the internal energy, $E_{I}$, i.e. viscous dissipation. $\varepsilon$ denotes viscous dissipation at the subgrid scales, i.e.

$$
\varepsilon=\nu\left\langle\frac{\partial u_{i, S G S}^{\prime}}{\partial x_{j}} \frac{\partial u_{i, S G S}^{\prime}}{\partial x_{j}}\right\rangle, \quad k_{S G S}=\frac{1}{2}\left\langle u_{i, S G S}^{\prime} u_{i, S G S}^{\prime}\right\rangle
$$

In steady RANS using an eddy-viscosity model the right circle vanishes, and most of the energy flow goes from $K$ to $k_{T}$ and only a small fraction directly from $K$ to $E_{I}$ (assuming low Mach number flow). In a well-resolved LES, the energy transfers from $K$ to $k_{T}$ and the transfer from $K$ to $E_{I}$ is negligible. In this case, the flow of kinetic energy goes from $K$ to $k_{r e s}$, and from $k_{r e s}$ to $k_{T}$, and then to $E_{I}$. Only a small fraction goes from $k_{r e s}$ to $E_{I}$.

From the discussion above in relation to Fig. 20, it is clear that an indicator of how well the flow is resolved is a comparison of the modelled dissipation from $K$ and $k_{r e s}$. The modelled dissipation in the $k_{r e s}$ equation is taken as $\varepsilon_{S G S}^{\prime}$, see Eq. 9. Figure 21 presents $\varepsilon_{S G S}$ and $\varepsilon_{S G S}^{\prime}$. As expected, the SGS dissipation due to the time-averaged flow dominates in the URANS region $\left(y^{+}<125\right)$ near the wall, see Fig. 21a. Further away from the wall 
(160 $\leq y^{+} \leq 200$, depending on the resolution), the $\varepsilon_{S G S}^{\prime} / \varepsilon_{S G S}$ ratio becomes larger than one half and is, for $y^{+}>800$, larger than 0.9 for all cases. It can also be seen in Fig. 21a that, the higher resolution, the higher the $\varepsilon_{S G S}^{\prime} / \varepsilon_{S G S}$ ratio; however, when $\varepsilon_{S G S}^{\prime}$ is not normalized with $\varepsilon_{S G S}$ (Fig. 21b), the picture is reversed, i.e. coarse resolution gives large $\varepsilon_{S G S}^{\prime}$. The results in Fig. 21b are partly explained by the high turbulent viscosity for the coarse resolutions (Fig. 6) and partly by higher turbulent resolved fluctuations. The correct approach is to present $\varepsilon_{S G S}^{\prime}$ in normalized form (Fig. 21a) because, in this way, the influence of the background flow is minimized; the $\varepsilon_{S G S}^{\prime}$ presented in Fig. 21b are biased by different mean flows, resolved turbulent fluctuations and SGS viscosities.

\section{Conclusions}

Five different grid resolutions have been investigated: Baseline $(\Delta x, \Delta z)$, two cases with a refined grid $(0.5 \Delta x$ and $0.5 \Delta z)$ and two cases with a coarsened grid $(2 \Delta z$ and $2 \Delta z)$. The resolution in the streamwise direction lies in the interval $200 \leq x^{+} \leq 800$ and $5 \leq \delta / \Delta x \leq 20$ and in the spanwise direction $100 \leq z^{+} \leq 400$ and $10 \leq \delta / \Delta z \leq 40$.

From the two-point correlations we find by how many cells the largest scales are resolved. This is very useful information. It is up to the researcher/engineer to decide how many cells he/she deems necessary, but the 
recommended minimum for a coarse LES should be at least four cells, preferably more than double that. Note that also eight cells is usually very far from a well-resolved LES. The two-point correlations presented in this work reveal that the resolutions for Cases $2 \Delta x$ and $2 \Delta z$ are much too coarse. Case $2 \Delta z$ yields almost zero two-point correlation at a separation distance of two cells. It is suggested that two-point correlations in the poorest resolved direction (the spanwise direction in the present work) are suitable for estimating the resolution.

Pope (2004) suggests that if the ratio of the resolved turbulent kinetic energy to the total (i.e. the resolved plus the modelled) is larger than $80 \%$, the LES simulation is well-resolved. The present work indicates that this is not a good measure because, for all five cases, this ratio is larger than $85 \%$.

One-dimensional energy spectra obtained from the two-point correlations have been presented. It is concluded that they are not useful for estimating the resolution.

It is found that the resolved streamwise fluctuation and the resolved turbulent kinetic energy increase, and that the correlation coefficient, $\left\langle-u^{\prime} v^{\prime}\right\rangle /\left(u_{r m s} v_{r m s}\right)$, decreases as the grid is coarsened. These phenomena are coupled to each other, since the time-averaged flow must satisfy a linear variation of the total shear stress. The correlation between $u^{\prime}$ and $v^{\prime}$ is weakened when the grid is coarsened, and the equations compensate for this by increasing the 
product of $u_{r m s}$ and $v_{r m s}$.

The spanwise resolved fluctuation, $w_{r m s}$, and the wall-normal one behave differently when the grid is refined and coarsened. Their behavior is explained by their largest source terms, the pressure velocity terms, $-2\left\langle w^{\prime} \partial p^{\prime} / \partial z\right\rangle$ $\left(w_{r m s}^{2}\right.$ equation) and $-2\left\langle v^{\prime} \partial p^{\prime} / \partial y\right\rangle\left(v_{r m s}^{2}\right.$ equation). Hence, it is seems that it is vital to accurately capture the interaction of the fluctuating velocities and pressure. Possibly some work should be directed towards development of discretization schemes which accurately treat the pressure-velocity coupling. Presumably, iterative solvers which treat pressure and velocities in a segregated manner (such as pressure correction schemes and fractional step techniques) are not optimal for accurate treatment of the velocity-pressure coupling; fully coupled solvers could be more accurate. Perhaps staggered grids could be more accurate than collocated arrangement since they avoid the Rhie-Chow interpolation. In fractional-step methods - as in the present method - the Rhie-Chow dissipation term is not added explicitly. It is, however, present implicitly, since the pressure gradient after the momentum equations have been solved is first subtracted from the velocity vector at the cell center and then, after the pressure Poisson equation has been solved, it is added to the velocity vector at the cell faces.

It is commonly assumed that the SGS dissipation takes place at the highest wavenumbers. It is found that this is not true; the largest dissipation 
takes place at rather small wavenumbers. One interpretation is that the effective filter width is larger than the grid spacing. As a result the velocity gradients are inaccurately computed for scales close to cut-off.

Consider the spanwise direction. Since the flow is homogeneous (i.e. periodic) in this direction, the spectral spanwise component of the spanwise viscous dissipation, $\varepsilon_{w z}$, can, according to the theory be computed from $\varepsilon_{w z}\left(\kappa_{z}\right)=\kappa_{z}^{2} E_{w w}$. It is found that this is different from the viscous dissipation component, $\varepsilon_{w z, F V}$, computed from the definition using finite volume discretization. The discrepancy arises because the derivative $\partial w^{\prime} / \partial z$ appearing in $\varepsilon_{w z}$ is computed analytically, whereas $\varepsilon_{w z, F V}$ is obtained in the finite volume procedure by computing the derivative $\partial w^{\prime} / \partial z$ numerically. Thus, the difference between $\varepsilon_{w z, F V}$ and $\varepsilon_{w z}$ is a measure of the inaccuracy of finite volume methods. The spectral difference is largest at the highest wavenumbers, because the numerical errors when computing these derivatives are the largest.

The final part of the paper investigates the ratio of the SGS dissipation due to resolved fluctuating strain rates to the total, i.e $\varepsilon_{S G S}^{\prime} / \varepsilon_{S G S}$. It is found that, in the LES region, this ratio lies between 0.5 and 0.9 . It decreases when the grid is coarsened. It can be noted that in homogeneous decaying turbulence, this ratio is by definition equal to one which indicates that it may not be an optimal parameter for estimating LES resolution. 


\section{Acknowledgments}

This work was financed by the DESider project (Detached Eddy Simulation for Industrial Aerodynamics) which was a collaboration between Alenia, ANSYSAEA, Chalmers University, CNRS-Lille, Dassault, DLR, EADS Military Aircraft, EUROCOPTER Germany, EDF, FOI-FFA, IMFT, Imperial College London, NLR, NTS, NUMECA, ONERA, TU Berlin, and UMIST. The project was funded by the European Community represented by the CEC, Research Directorate-General, in the 6th Framework Programme, under Contract No. AST3-CT-2003-502842.

Financial support by SNIC (Swedish National Infrastructure for Computing) for computer time at C3SE (Chalmers Center for Computational Science and Engineering) is gratefully acknowledged.

\section{References}

Balaras, E., Benocci, C., Piomelli, U., 1996. Two-layer approximate boundary conditions for large-eddy simulations. AIAA Journal 34, 1111-1119.

Cabot, W., 1995. Large eddy simulations with wall models. In: Annual Research Briefs. Center for Turbulent Research, Stanford Univ./NASA Ames Research Center, pp. $41-50$.

Cabot, W., Moin, P., 1999. Approximate wall boundary conditions in the largeeddy simulations of high Reynolds number flow. Flow, Turbulence and Combustion 63, 269-201. 
Davidson, L., Billson, M., 2006. Hybrid LES/RANS using synthesized turbulence for forcing at the interface. International Journal of Heat and Fluid Flow 27 (6), $1028-1042$.

Davidson, L., Peng, S.-H., 2003. Hybrid LES-RANS: A one-equation SGS model combined with a $k-\omega$ model for predicting recirculating flows. International Journal for Numerical Methods in Fluids 43, 1003-1018.

Emvin, P., 1997. The full multigrid method applied to turbulent flow in ventilated enclosures using structured and unstructured grids. Ph.D. thesis, Dept. of Thermo and Fluid Dynamics, Chalmers University of Technology, Göteborg.

Hamba, F., 2003. A hybrid RANS/LES simulation of turbulent channel flow. Theoretical and Computational Fluid Dynamics 16, 387-403.

Hinze, J., 1975. Turbulence, 2nd Edition. McGraw-Hill, New York.

Hoyas, S., Jiménez, J., 2006. Scaling of the velocity fluctuations in turbulent channels up to $R e_{\tau}=2003$. Physics of Fluids A 18 (011702).

Keating, A., Piomelli, U., 2006. 6 a dynamic stochastic forcing method as a walllayer method model for large-eddy simulation. Physics of Fluids A 7 (12), 1-24.

Moin, P., Kim, J., 1982. Numerical investigation of turbulent channel flow. Journal of Fluid Mechanics 118, 341-377.

Moser, R., Kim, J., Mansour, N., 1999. Direct numerical simulation of turbulent channel flow up to $R e_{\tau}=590$. Physics of Fluids 11, 943-945. 
Piomelli, U., 1993. High Reynolds number calculations using the dynamic subgridscale stress model. Physics of Fluids A 5, 1484-1490.

Piomelli, U., Balaras, E., Pasinato, H., Squire, K., Spalart, P., 2003. The innerouter layer interface in large-eddy simulations with wall-layer models. International Journal of Heat and Fluid Flow 24, 538-550.

Piomelli, U., Chasnov, J., 1996. Large-eddy simulations: Theory and applications. In: Henningson, D., Hallbäck, M., Alfredsson, H., Johansson, A. (Eds.), Transition and Turbulence Modelling. Kluwer Academic Publishers, Dordrecht, pp. 269-336.

Pope, S., 2004. Ten questions concerning the large-eddy simulations of turbulent flows. New Journal of Physics 6 (35), 1-24.

Robinson, S., 1991. Coherent motions in the turbulent boundary layer. Annual Review of Fluid Mechanics 23, 601-639.

Spalart, P., Jou, W.-H., Strelets, M., Allmaras, S., 1997. Comments on the feasability of LES for wings and on a hybrid RANS/LES approach. In: Liu, C., Liu, Z. (Eds.), Advances in LES/DNS, First Int. conf. on DNS/LES. Greyden Press, Louisiana Tech University.

Temmerman, L., Leschziner, M., Hanjalić, K., 2002. A-priori studies of near-wall RANS model within a hybrid LES/RANS scheme. In: Rodi, W., Fueyo, N. 
(Eds.), Engineering Turbulence Modelling and Experiments 5. Elsevier, pp. 317326.

Tessicini, F., Li, N., Leschziner, M., 2007. Large-eddy simulation of threedimensional flow around a hill-shaped obstruction with a zonal near-wall approximation. International Journal of Heat and Fluid Flow 28 (5), 894-908.

Travin, A., Shur, M., Strelets, M., Spalart, P., 2000. Detached-eddy simulations past a circular cylinder. Flow Turbulence and Combustion 63 (1/4), 293-313.

Tucker, P., Davidson, L., 2004. Zonal k-l based large eddy simulation. Computers \& Fluids 33 (2), 267-287.

Wang, M., Moin, P., 2002. Dynamic wall modelling for large eddy simulation of complex turbulent flows. Physics of Fluids 14 (7), 2043-2051. 